\title{
Alternativas de terapia neoadyuvante en el manejo del paciente con cáncer de ovario recurrente, a propósito del artículo: Rol de la citorreducción secundaria en cáncer de ovario recurrente
}

\author{
Pablo Salomón Montes-Arcón. ${ }^{1}$
}

Luego de realizar la lectura detallada del artículo "Rol de la citorreducción secundaria en cáncer de ovario recurrente", es de conocimiento médico que la enfermedad neoplásica del ovario representa un reto diagnóstico y terapéutico, principalmente porque su diagnóstico es tardío; siendo la octava causa más común de muerte por cáncer en las mujeres y la segunda causa de mortalidad por cáncer ginecológico a nivel mundial (1).

Desafortunadamente, la enfermedad neoplásica recurrente de ovario se desarrolla en un porcentaje considerable de pacientes, estimada en más del $80 \%$ de las mujeres y con tasas de supervivencia libre de enfermedad a 10 años en pacientes con enfermedad por debajo del $15 \%$ (2). Cerca de un $22 \%$ de las recaídas se presentan durante los primeros 6 meses, mientras que la mayoría de las pacientes presentan recaída sensible al platino, con un intervalo de más de 6 meses después de la quimioterapia basada en platino (3). Actualmente, la cirugía y la quimioterapia son la piedra angular del manejo de estas pacientes, siendo el tamaño tumoral residual posquirúrgico el factor pronóstico más relevante y el sitio principal de

${ }^{1}$ Médico general, Universidad del Magdalena. Residente de primer año, postgrado de Patología, Universidad de Cartagena, Bolívar, Colombia. Correo para correspondencia: pmontesarcon@gmail.com

Forma de citar este artículo: Montes-Arcón PS. Alternativas de terapia neoadyuvante en el manejo del paciente con cáncer de ovario recurrente, a propósito del artículo: Rol de la citorreducción secundaria en cáncer de ovario recurrente. Rev Obstet Ginecol Venez. 2022; 82(1): 136-137. https://doi.org/10.51288/00820117 afección residual es el peritoneo, con incidencias de hasta el $75 \%$ en sujetos con 0 enfermedad residual (4).

En pacientes que presentan cáncer de ovario recurrente a peritoneo, existen actualmente alternativas terapéuticas que mejoran considerablemente la sobrevida de quienes son sometidos a estas, tales como la quimioterapia intraperitoneal intraoperatoria hipertérmica (HIPEC) y la quimioterapia en aerosol intraperitoneal presurizada (PIPAC) (5).

La quimioterapia intraperitoneal intraoperatoria hipertérmica (HIPEC) es una técnica consistente en administrar un agente quimioterapéutico, con la perfusión de una solución calentada del medicamento en todo el espacio peritoneal, su objetivo es apuntar a los residuos de enfermedad después de la cirugía citorreductora (CRS), al actuar directamente sobre las células cancerosas presentes en la superficie peritoneal mediante citotoxicidad directa por calor y por la sinergia con medicamentos antimitóticos tales como cisplatino, paclitaxel, oxaliplatino y mitomicina; con lo que se aprovecha además que la barrera hematoperitoneal limita la absorción sistémica del agente quimioterapéutico $\mathrm{y}$, por lo tanto, reduce sus efectos secundarios y toxicidad (6). En la actualidad, su utilidad se encuentra, principalmente, en pacientes con cáncer de ovario primario y recidivante, estadio III y IV, con probabilidad de resección total, sin embargo, no se considera como tratamiento de primera línea (7). 
La quimioterapia en aerosol intraperitoneal presurizada (PIPAC) es una técnica novedosa que consiste en la administración, por vía laparoscópica, de quimioterapia (cisplatino, paclitaxel, oxaliplatino y mitomicina) mediante aerosol a alta presión de $12 \mathrm{~mm}$ de $\mathrm{Hg}$, con una mayor penetración del medicamento y mejor distribución usando dosis más bajas que las habituales en la HIPEC, lo que genera unos menores efectos secundarios; sin embargo, la PIPAC se administra en tejido tumoral no resecado, mientras que la HIPEC sobre el lecho tumoral y en residuos tumorales de escasa cuantía (8). Sus indicaciones actuales son pacientes con enfermedad irresecable, paciente con metástasis única, paciente no elegible para CRS extensa y/o HIPEC, ascitis recurrente, hallazgos histológicos poco favorables (células en anillo de sello, carcinoma de células claras, cáncer de ovario indiferenciado) y pacientes en manejo con terapias de segunda y tercera línea (9).

Por lo anterior, estas alternativas en la actualidad son más que interesantes como terapias en aquellos pacientes con cáncer de ovario recurrente, en algunos como posibilidad curativa (HIPEC) y en otros como manejo paliativo (PIPAC); sin embargo, se requieren más estudios para determinar sus indicaciones puntuales. Razón suficiente para indagar la posibilidad de su estudio e implementación en América latina dada una incidencia no despreciable de 9,2 y una tasa de mortalidad de 7,3 por 100000 mujeres, con lo que se beneficiaría a aquellas pacientes susceptibles a estos manejos $(6,10)$.

\section{REFERENCIAS}

1. LaraE, Calderaro Di Ruggiero F. Rol de la citorreducción secundaria en cáncer de ovario recurrente. Rev Obstet Ginecol Venez. 2021; 81(2):155-161 https://doi. org/10.51288/00810209

2. Berek JS, Kehoe ST, Kumar L, Friedlander M. Cancer of the ovary, fallopian tube, and peritoneum. Int $\mathrm{J}$ Gynaecol Obstet. 2018; 143(Suppl2):59-78. doi: 10.1002/ijgo.12614.

Vol. 82, $\mathrm{N}^{\mathrm{o}}$ 1, enero 2022.
3. du Bois A, Reuss A, Pujade-Lauraine E, Harter P, Ray-Coquard I, Pfisterer J. Role of surgical outcome as prognostic factor in advanced epithelial ovarian cancer: a combined exploratory analysis of 3 prospectively randomized phase 3 multicenter trials: by the Arbeitsgemeinschaft Gynaekologische Onkologie Studiengruppe Ovarialkarzinom (AGO-OVAR) and the Groupe d'Investigateurs Nationaux Pour les Etudes des Cancers de l'Ovaire (GINECO). Cancer. 2009; 115(6):1234-1244. doi: 10.1002/cncr.24149.

4. Bristow RE, Tomacruz RS, Armstrong DK, Trimble EL, Montz FJ. Survival effect of maximal cytoreductive surgery for advanced ovarian carcinoma during the platinum era: a meta-analysis. J Clin Oncol. 2002; 20(5):1248-1259. doi: 10.1200/JCO.2002.20.5.1248.

5. Leebmann $\mathrm{H}$, Piso P. PIPAC und HIPEC konkurrierende oder ergänzende Therapieverfahren bei peritonealen metastasen. Der Chirurg. 2018; 89(9):693698. doi: 10.1007/s00104-018-0666-6.

6. Mishra M, Singh N, Ghatage P. Past, present, and future of hyperthermic intraperitoneal chemotherapy (HIPEC) in ovarian cancer. Cureus. 2021; 13(6):e15563. doi:10.7759/cureus. 15563

7. Fagotti A, Costantini B, Petrillo M, Vizzielli G, Fanfani F, Margariti PA, et al. Cytoreductive surgery plus HIPEC in platinum-sensitive recurrent ovarian cancer patients: a case-control study on survival in patients with two year follow-up. Gynecol Oncol. 2012; 127(3):502-505. doi: 10.1016/j.ygyno.2012.09.020.

8. Alonso-Gómez J, Silvestre-Rodríguez J, BermejoGuillén MI, Artíles-Armas M, Marchena-Gómez J. Primer tratamiento PIPAC en un hospital público español. Una novedosa técnica para el tratamiento de la carcinomatosis. Cir Esp. 2019; 97(3):177-182. DOI: 10.1016/j.ciresp.2018.08.007

9. Alyami M, Hübner M, Grass F, Bakrin N, Villeneuve L, Laplace N, et al. Pressurised intraperitoneal aerosol chemotherapy: rationale, evidence, and potential indications. Lancet Oncol. 2019; 20(7):e368-e377. doi: 10.1016/S1470-2045(19)30318-3.

10. Martínez-Ospina AP, Porras-Ramírez A, Rico-Mendoza A. Epidemiologia de cáncer de ovario colombia 20092016. Rev Chil Obstet Ginecol. 2019; 84(6):480-489. http://dx.doi.org/10.4067/S0717-75262019000600480

Recibido 18 de agosto de 2021 Aprobado 15 de noviembre de 2021 